\title{
Can the gamma-ray source 3EG J2033+4118 be produced by the stellar system Cygnus OB2 No. 5?
}

\author{
P. Benaglia ${ }^{1, \star}$, G. E. Romero ${ }^{1, \star}$, I. R. Stevens ${ }^{2}$, and D. F. Torres ${ }^{1, \star \star}$ \\ 1 Instituto Argentino de Radioastronomía, C.C.5, (1894) Villa Elisa, Bs. As., Argentina \\ 2 School of Physics \& Astronomy, University of Birmingham, Edgbaston, Birmingham B15 2TT, UK
}

Received 3 October 2000 / Accepted 13 November 2000

\begin{abstract}
We discuss the possibility that the stellar system Cyg OB2 No. 5 can be a gamma-ray source in the light of recent EGRET and radio data. This system is formed by an O7 Ia + Ofpe/WN9 contact binary. A third star, probably a B0 V star also associated with the system, is located at $\sim 1700$ AU from the primary. We estimate the expected gamma-ray luminosity from the colliding winds region, the terminal shock of the wind, and the unstable zone at the base of the wind, and conclude that, under very reasonable assumptions, Cyg OB2 No. 5 can generate about a half of the gamma-ray flux detected from the positionally coincident source 3EG J2033+4118. We suggest, then, that other O stars belonging to the association, also placed within the $95 \%$ probability EGRET location contour, could contribute to the observed gamma-ray flux.
\end{abstract}

Key words. gamma-rays: theory - gamma-rays: observations - stars: early-type - stars: winds, outflows

\section{Introduction}

High-energy emission from early-type stars has been often discussed from a theoretical point of view (e.g. Cassé \& Paul 1980; Völk \& Forman 1982; White 1985; Chen \& White 1991; White \& Chen 1992; Eichler \& Usov 1993). The observational evidence for its existence, however, has remained rather elusive till now.

The recent publication of the Third EGRET (3EG) Catalog by Hartman et al. (1999) provides new information that can be very useful for the identification of possible gamma-ray emitting stellar systems. There are 170 unidentified sources in the new catalog, and approximately half of them are concentrated near the galactic plane (e.g. Gehrels et al. 2000). Romero et al. (1999) have studied the positional coincidence between 3EG sources and different types of galactic objects such as supernova remnants (SNRs), OB star associations (usually considered as tracers of pulsar concentrations), and massive stars like WolfRayet (WR) and Of stars, which have very strong stellar winds. They have found that 10 gamma-ray sources in the 3 EG catalog have WR or Of stars within their $95 \%$ confidence location contours. The probability of this being a mere effect of chance is in the range $10^{-2}-10^{-3}$. Although such probabilities are not as low as those found for SNRs and OB associations, they are suggestive enough as

Send offprint requests to: P. Benaglia

* Member of CONICET.

$\star \star$ Fellow of CONICET. to encourage an a posteriori analysis of some interesting candidates. In particular, Romero et al. (1999) mentioned the possibility that the system Cyg OB2 No. 5 could be physically linked to the source 3EG J2033+4118, and hence that it could be the first stellar system to be detected at gamma-rays, involving other than WR stars.

In this paper we discuss whether Cyg OB2 No. 5 can actually generate the observed gamma-ray flux and which are the relevant radiation mechanisms for this system at high energies. Our results will be relevant not only for this particular system but also for other interacting binaries like WR 140 (Romero et al. 1999; Zhekov \& Skinner 2000), which is also positionally coincident with a gamma-ray source, and WR 147, for which there are detailed radio observations (Contreras \& Rodríguez 1999). The interest of stellar systems as potential gamma-ray sources has been recently reinforced by the suggested association of $3 \mathrm{EG}$ J1824-1514 with an X-ray binary by Paredes et al. (2000).

We shall begin our study by reviewing the main characteristics of Cyg OB2 No. 5.

\section{The stellar system Cyg OB2 No. 5}

Cyg OB2 No. 5 (also known as V729 Cyg, BD +404220) is an evolved contact binary system with a period of 6.6 days (Hall 1974) located at a distance of $\sim 1.8 \mathrm{kpc}$ (e.g. Waldron et al. 1998). The primary star in the system is a very massive star classified by Walborn (1973) as O7 Ianfp, although it should be taken into account that 
Table 1. Stellar parameters

\begin{tabular}{|c|c|c|c|c|c|c|c|c|}
\hline Star & Sp. Class. & $\log T_{\text {eff }}$ & $\begin{array}{c}R_{*} \\
\left(R_{\odot}\right)\end{array}$ & $\log \left(L / L_{\odot}\right)$ & $\begin{array}{l}B_{*} \\
(\mathrm{G})\end{array}$ & $\begin{array}{c}\dot{M} \\
\left(10^{-5} M_{\odot} / \mathrm{yr}\right)\end{array}$ & $\begin{array}{c}v_{\infty} \\
\left(\mathrm{km} \mathrm{s}^{-1}\right)\end{array}$ & $\begin{array}{c}V_{\text {rot }} \\
\left(\mathrm{km} \mathrm{s}^{-1}\right)\end{array}$ \\
\hline Primary & O7 Ianfp ${ }^{(a)}$ & $4.6^{(\mathrm{c})}$ & $34^{(\mathrm{d})}$ & $5.984^{(\mathrm{c})}$ & 2 & $2.5^{(\mathrm{f})}$ & $2200^{(\mathrm{d})}$ & $180^{(\mathrm{d})}$ \\
\hline Secondary & B0 $V^{(b)}$ & $4.5^{(\mathrm{c})}$ & $8.3^{(\mathrm{c})}$ & $4.881^{(\mathrm{c})}$ & 1 & $0.2^{(\mathrm{f})}$ & $1000^{(\mathrm{b})}$ & $150^{(\mathrm{e})}$ \\
\hline
\end{tabular}

(a): Walborn (1973); (b): Contreras et al. (1997); (c) Vacca et al. (1996); (d): Bieging et al. (1989);

(e): Uesugi \& Fukuda (1982); (f): see text.

a sizable fraction of the line emission arises in the interaction region between the two stars and is not intrinsic to the stars themselves. Most of the spectral features that justify the "nfp" tag are actually emission lines that form, to a large extent, in the wind interaction region of the close binary.

Bohannan \& Conti (1976) also classified the secondary star as an Of star with similar luminosity, since each star seems to contribute to the He II $\lambda 4686$ emission. This result has been recently reconsidered by Rauw et al. (1999), who have presented a detailed analysis of an extensive set of spectroscopic observations finding a new orbital solution. These authors showed that the observed properties can be explained if the secondary is a transition Ofpe/WN9 star, with a much slower terminal wind velocity than the primary. Consequently, at some distance from the system, the wind properties are basically determined by the primary, with a terminal velocity of $v_{\infty} \sim 2200 \mathrm{~km} \mathrm{~s}^{-1}$ (Bieging et al. 1989).

Cyg OB2 No. 5 is well known as a rather powerful and variable radio source (Abbott et al. 1981; Persi et al. 1985, 1990; Miralles et al. 1994). The radio source seems to switch between states of high and low emission with a period of about 7 years. Miralles et al. (1994) detected, in addition to a strong source coincident with the contact binary, a weak radio component, possibly nonthermal, located at $\sim 1$ arcsec towards the NE of the binary system. They proposed that the radio variability is produced by relativistic particles accelerated in the wind. The weak radio component, however, is too far from the binary system as to be the radio counterpart of the secondary. In its approximate position, a star of magnitude 13-14 has been previously reported by Herbig (1967).

Recently, Contreras et al. (1997) have obtained accurate optical (HIPPARCOS and Roque de los Muchachos) and radio (VLA) measurements that clearly show that the weak radio source is nonthermal and does not coincide with the optical position of the star detected by Herbig (1967). This star, which seems to be a B0 V star, is a third component of the system, with a long period. The fact that the weak radio source is located between the contact binary and the B0 V star, closer to the latter, was interpreted by Contreras et al. (1997) as the effect of the particle acceleration in the region of colliding winds.
In this region, electrons locally accelerated radiate synchrotron emission, which is detected at centimeter wavelengths as the weak radio component.

The system Cyg OB2 No. 5, then, seems to be formed by three stars: an O7 Ia + Ofpe/WN9 contact binary and a B0 $\mathrm{V}$ star in a larger orbit. When evaluating the gamma-ray production in Cyg OB2 No. 5, we shall consider the contact binary, whose winds are dominated by the O7 Ia star, as the primary object in the presumed gamma-ray emitting system, and the B0 $\mathrm{V}$ star as the secondary.

In Table 1 we summarize the stellar parameters adopted in this paper. The mass loss rate of the primary has been derived from $\mathrm{H} \alpha$, infrared and radio observations, giving values in the range 1.9 to $2.610^{-5} M_{\odot} \mathrm{yr}^{-1}$ (e.g. Leitherer et al. 1982; Persi et al. 1990). Contreras et al. (1997) propose an extreme $\dot{M}=5.510^{-5} M_{\odot} \mathrm{yr}^{-1}$ that should be considered rather as an upper limit. In our present work we adopt an average value of $2.510^{-5} M_{\odot} \mathrm{yr}^{-1}$. The mass loss rate for the secondary results from that adopted for the primary and geometrical considerations on the location of the colliding wind region (see Sect. 4.1). The effective temperatures and the stellar luminosities are taken from Vacca et al. (1996). There are no direct measurements of the stellar magnetic fields, although they are usually thought to be of the order of a few Gauss (e.g. Maheswaran \& Cassinelli 1992). We have adopted values of 2 and $1 \mathrm{G}$ for the primary and the secondary, respectively. A larger field would produce a turnover in the synchrotron emission of the primary at a few $\mathrm{GHz}$ that is not observed, whereas a smaller value would shift this turnover to hundreds of $\mathrm{GHz}$, resulting in an excess of non-thermal emission at high frequencies. Our assumptions are in agreement with White \& Chen (1992) and allow a fair description of the observed radio spectrum for the primary in terms of White's (1985) model (see Sect. 4.3).

\section{The gamma-ray source $3 E G \mathrm{~J} 2033+4118$}

According to the 3EG catalog (Hartman et al. 1999), the best estimated position of $3 \mathrm{EG} \mathrm{J} 2033+4118$ is at $(l, b) \approx\left(80.27^{\circ}, 0.73^{\circ}\right)$, i.e. at 0.27 degrees from $\mathrm{Cyg}$ OB2 No. 5 (see Fig. 1). The summed EGRET flux 


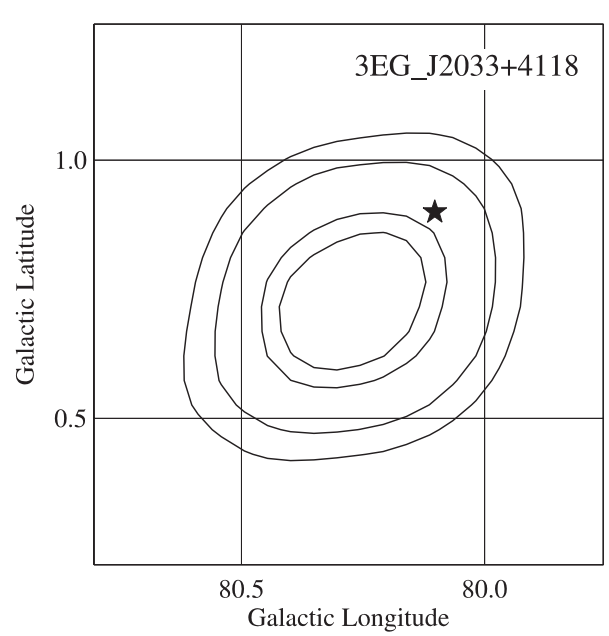

Fig. 1. Location map of the gamma-ray source 3EG J2033+4118. The contours represent 50\%, 68\%, 95\% and $98 \%$ statistical probability that the source lies within that contour. The position of the stellar system Cyg OB2 No. 5 is indicated with a star symbol

$(E>100 \mathrm{MeV})$ for cycles from 1 to 4 (i.e. for the time span 1991-1995) is $(73.0 \pm 6.7) 10^{-8} \mathrm{ph} \mathrm{cm}^{-2} \mathrm{~s}^{-1}$. If we assume that the source is at the distance of Cyg OB2 No. $5(1.8 \mathrm{kpc})$, an integrated gamma-ray luminosity of $\sim 2.410^{35} \mathrm{erg} \mathrm{s}^{-1}$ is implied for the EGRET energy range $(100 \mathrm{MeV}-20 \mathrm{GeV})$. The photon spectral index is $\Gamma=1.96 \pm 0.10\left(N(E) \propto E^{-\Gamma}\right)$. For all calculations we assume $\Gamma=2$.

In order to estimate the degree of variability of the gamma-ray emission we have adopted the method used by Zhang et al. (2000), Romero et al. (2000) and Torres et al. (2000). A variability index $I$ is defined such that $I=\mu_{\mathrm{s}} /<\mu>_{\mathrm{p}}$, where $\left.\mu_{\mathrm{s}}=100 \sigma<F\right\rangle^{-1}$ is the fluctuation index of the gamma-ray source and $\langle\mu\rangle_{\mathrm{p}}$ is the averaged fluctuation index for all known gamma-ray pulsars, which are usually considered as a non-variable population. $F$ is the flux and $\sigma$ its standard deviation. A variable source, then, should satisfy that $I>1$. For 3 EG J2033+4118 we obtain $I=1.3$, so, taken into account the errors involved $(1 \sigma=0.5)$, the source cannot be classified as clearly variable, at least over the timescale of the observations ( $\sim 4 \mathrm{yr})$. This do not preclude that the source could experience variability on longer timescales, for instance with a period similar to what is observed at radio frequencies in Cyg OB No. 5. (i.e., $\sim 7$ yr). We shall have to wait until the next generation of gamma-ray satellites to get a confident evaluation of the temporal behaviour of this source.

\section{Gamma-ray production in Cyg OB2 No. 5}

The main mechanisms that can generate gamma rays at energies $E>100 \mathrm{MeV}$ in a stellar environment are inverse Compton (IC) scattering, relativistic bremsstrahlung, and $\pi^{0}$-decay from hadronic interactions. All these mechanisms require the existence of a relativistic population of charged particles (electrons or positrons in the first two cases, protons or ions in the latter). Effective particle acceleration up to high energies requires, in turn, the mediation of strong shock fronts (e.g. Bell 1978a,b; Blandford \& Ostriker 1978; Blandford 1980; Drury 1983): the particles are scattered by magnetic irregularities carried by the converging flow on either side of the shock wave, leading to a first-order Fermi process that results in a power-law spectrum of relativistic particles.

In a system such as Cyg OB2 No. 5 there are several places where strong shocks can be formed. In particular, strong shocks are expected in the colliding winds region located at some point between the primary and secondary stars (i.e. between the contact binary and the B0 V star), at the point where the momentum flux of the primary (and stronger) wind decline to the value of interstellar pressure (i.e. the terminal shock), and at the base of the wind where line-driven instabilities are thought to occur. These shocks locally re-accelerate the particles making them energetic enough as to generate the gamma-rays. We shall discuss below in major detail the possible gamma-ray production in each of the mentioned regions.

\subsection{Region of colliding winds}

The supersonic winds from the primary and secondary stars flow nearly radially out to the shocks. The geometry of the region where the winds collide is described by Eichler \& Usov (1993) in terms of the star separation $D$ and the parameter $\eta=\dot{M}_{2} v_{\infty, 2} / \dot{M}_{1} v_{\infty, 1}$ as:

$$
r_{1}=\frac{1}{1+\eta^{1 / 2}} D, \quad r_{2}=\frac{\eta^{1 / 2}}{1+\eta^{1 / 2}} D
$$

Here, a subscript "1" stands for the primary whereas the subscript " 2 " denotes the secondary. $\dot{M}$ is the mass loss rate, $v_{\infty}$ is the wind terminal velocity, and $r$ the distance from the star to the colliding winds region. Since $\eta<1$, this region will be closer to the secondary.

Eichler \& Usov (1993) have shown that particles locally accelerated at the strong shock front formed in the wind collision can generate observable synchrotron radio emission. Contreras et al. (1997) have identified the weak non-thermal radio source in Cyg OB2 No. 5 with this emission. The same population of relativistic electrons that emits the synchrotron radiation should produce high-energy gamma-rays through IC interactions with the UV photons from the secondary. The frequency of the IC photons is given by $\nu_{\mathrm{IC}}=4 / 3 \gamma^{2} \nu_{*}$, where $\gamma$ is the Lorentz factor of the relativistic electrons $\left(\gamma \sim 3.510^{3}\right.$ for electrons that produce synchrotron photons of $5 \mathrm{GHz}$ in a field of $\sim 10^{-4} \mathrm{G}$ ) and $\nu_{*}$ is the frequency of the seed stellar photons. The ratio of synchrotron to IC luminosity is in this case (e.g. White \& Chen 1995):

$\frac{L_{\mathrm{syn}}}{L_{\mathrm{IC}}}=840 \frac{B_{\mathrm{s}}^{2} r_{2}^{2}}{L_{2}}$ 
where $B_{\mathrm{S}}$ is the magnetic field in Gauss, $r_{2}$ the distance to the secondary in $\mathrm{AU}$, and $L_{2}$ is its luminosity in $L_{\odot}$ units.

From geometric considerations, Contreras et al. (1997) derive $\eta \sim 0.04$, and then $r_{2} \sim 300$ AU. The integrated synchrotron luminosity between the two observing frequencies of the nonthermal radio component is $L_{\text {syn }} \sim$ $4.510^{27} \mathrm{erg} \mathrm{s}^{-1}$. Taken into account that for a B0 V star the peak of the photon distribution is at $\nu_{*} \sim 210^{15} \mathrm{~Hz}$, we have that only electrons with Lorentz factors in the range $3.010^{3}-4.310^{4}$ will contribute to the IC gammaray flux in the EGRET energy range.

In order to estimate the total synchrotron luminosity of these particles, we must first evaluate the magnetic field at the colliding winds region. We shall assume that the external magnetic field of the star in the absence of stellar wind is dipole, and that in the presence of wind it obeys the standard $B \propto r^{-1}$ radial dependence for large $r$ given by Eichler \& Usov (1993):

$B \approx B_{*} \frac{V_{\text {rot }}}{v_{\infty}} \frac{R_{*}^{2}}{r_{\mathrm{A}} r}, \quad$ valid for $r>R_{*} \frac{v_{\infty}}{V_{\text {rot }}}$,

where $R_{*}$ is the stellar radius, $B_{*}$ the surface magnetic field, $V_{\text {rot }}$ the surface rotation velocity, and $r_{\mathrm{A}} \approx R_{*}$ the Alfvén radius. Using the parameters listed in Table 1 we find that in the colliding winds region the field is $B \sim 210^{-5} \mathrm{G}$, a value about a factor 10 larger than the average magnetic field in the standard ISM (Spitzer 1998). We shall adopt, taking into account the compression produced by the strong shock formed in the wind collision, a field $B_{\mathrm{s}}=10^{-4} \mathrm{G}$. Then, the integrated synchrotron luminosity of the electrons that contribute to the IC gammaray luminosity is $L_{\mathrm{syn}} \approx 510^{29} \mathrm{erg} \mathrm{s}^{-1}$. From Eq. (2), we obtain that the corresponding inverse Compton luminosity with seed photons from the secondary in the EGRET energy range is $\sim 510^{34} \mathrm{erg} \mathrm{s}^{-1}$. If we also consider the contribution from the photon field of the primary, we get a total IC luminosity of the colliding wind region of $\sim 810^{34} \mathrm{erg} \mathrm{s}^{-1}$.

Additional contributions from bremsstrahlung and hadron interactions can be expected from the colliding winds region. The density of particles in this region is (Eichler \& Usov 1993):

$$
\begin{aligned}
n \simeq & \frac{\dot{M}_{1}}{4 \pi r_{1}^{2} v_{\infty, 1} m_{\mathrm{p}} \mu} \\
\simeq & \frac{310^{9}}{\mu}\left(\frac{\dot{M}_{1}}{210^{-5} M_{\odot} / \mathrm{yr}}\right)\left(\frac{r_{1}}{10^{13} \mathrm{~cm}}\right)^{-2} \\
& \times\left(\frac{v_{\infty, 1}}{210^{8} \mathrm{~cm} / \mathrm{s}}\right)^{-1} \mathrm{~cm}^{-3} .
\end{aligned}
$$

Here, $m_{\mathrm{p}}$ is the proton mass and $\mu$ is the mean molecular weight of the gas, taken as 1.3. Using Eq. (4) and assuming again a compression factor of 4 for the post-shock region, we estimate a number density $n \simeq 2.910^{3} \mathrm{~cm}^{-3}$. This means that the total mass in the colliding winds region is $\sim 2.210^{-6} M_{\odot}$. In this calculation we have assumed a spherical geometry with a radius equal to the average value of the semi-axes in Contreras et al.'s (1997) 6-cm elliptical image of the secondary radio source.

The contribution from $\pi^{0}$-decays, consequently, should be very low, even if an important enhancement in the protonic cosmic ray energy density is occurring due to local reacceleration. We estimate a luminosity of $\sim 5.210^{24} \mathrm{erg} \mathrm{s}^{-1}$ for a cosmic ray enhancement of a factor 10, using Eq. (19) in Aharonian \& Atoyan (1996).

In the case of relativistic bremsstrahlung, which is produced in the electrostatic fields of local ions by the same electronic population that emits the observed synchrotron radiation, we have that the ratio of gamma-ray bremsstrahlung $\left(F_{\gamma}\right)$ to synchrotron radio $\left(S_{\mathrm{syn}}\right)$ fluxes is given by (see Pollock 1985):

$$
\begin{aligned}
R & =\frac{F_{\gamma}(>100 \mathrm{MeV})}{S_{\mathrm{syn}}} \\
& \simeq 1.410^{-13}\left(\frac{n}{\mathrm{~cm}^{-3}}\right)\left(\frac{B_{\mathrm{s}}}{\mu \mathrm{G}}\right)^{-3 / 2}\left(\frac{\nu}{\mathrm{Hz}}\right)^{1 / 2} \frac{\mathrm{cm}^{-2} \mathrm{~s}^{-1}}{\mathrm{Jy}}
\end{aligned}
$$

The above expression has been derived for a canonical power-law electron spectrum of index $p=2\left(N_{\mathrm{e}}(E) \propto\right.$ $\left.E^{-p}\right)$. Using the observed radio flux at $5 \mathrm{GHz}$, and the same physical parameters than in the case of the $\pi^{0}$-decays, we obtain a bremsstrahlung contribution of $\sim 3.410^{30} \mathrm{erg} \mathrm{s}^{-1}$. This value is more than a thousand times smaller than the contribution from the inverse Compton emission, that clearly dominates the high-energy radiation from this region.

We conclude that, according to the available radio observations, a significant part of the observed gammaray flux from $3 \mathrm{EG} \mathrm{J} 2033+4118$ might be produced in the colliding winds region of Cyg OB2 No. 5 .

\subsection{Terminal shock}

Particle acceleration and gamma-ray production in the shocked region at the boundary between a supersonic stellar wind and the surrounding medium has been discussed in detail by Cassé \& Paul (1980) and Völk \& Forman (1982). White (1985) showed that energetic particles from the star can be injected into the acceleration region despite the strong adiabatic losses. At the terminal shock, the charged particles are accelerated to a power law distribution with index 2. Observable gamma-ray radiation can be generated if there is a nearby source of photons to interact with the electrons or nearby clouds that might be "illuminated" by the relativistic protons. In the case of Cyg OB2 No. 5, if there is a contribution from the terminal shock to the total gamma-ray luminosity, it should come from the latter mechanism because there is no evidence of synchrotron emission from the terminal shock that reveals a particularly high energy density of relativistic electrons. On the contrary, there is evidence of several compact clouds in the region. Dobashi et al. $(1994,1996)$ have made an extensive study of molecular clouds in the Cygnus 
region. They have found 159 molecular clouds through large-scale ${ }^{13} \mathrm{CO}$ observations in the region. The mass spectrum of these clouds is found to be well approximated by a power law $\mathrm{d} N / \mathrm{d} M_{\mathrm{cl}} \propto M_{\mathrm{cl}}^{-1.6}$, for $M_{\mathrm{cl}}>100 M_{\odot}$. In the neighborhood of Cyg OB2 No. 5, four clouds with estimated masses of $>230,>6750,1530$, and $<240 M_{\odot}$ have been detected. The presence of many protostellar candidates detected by IRAS also indicates a large number of small, dense clouds. It is not unreasonable, then, to assume that at least one small cloud of, say, $100 M_{\odot}$ could be illuminated by protons locally accelerated in the terminal shock of the wind, at a few pc from the primary star. If the local density of cosmic rays is enhanced in a factor 10 from the value observed in the Earth vicinity (e.g. Dermer 1986), we can expect an integrated gammaray luminosity of $\sim 2.310^{32} \mathrm{erg} \mathrm{s}^{-1}$ in the EGRET range, with the conservative assumption that only a small cloud of $100 M_{\odot}$ is overtaken.

\subsection{Base of the wind}

The winds of $\mathrm{O}$ and $\mathrm{B}$ stars are radiatively driven by absorption in spectral lines and are prone to undergo instabilities that can grow up to form strong shocks at the base of the outflow (e.g. Lucy \& White 1980; Lucy 1982; Lamers \& Casinelli 1999). These shocks can accelerate particles to relativistic energies. If the particles are electrons, synchrotron and IC radiation can be produced. White (1985) have calculated the electron energy spectrum and the associated synchrotron spectrum, and have shown that these spectra can be quite different from the naive pure powerlaw case. In particular, the wind synchrotron spectrum rises with frequency as $S_{\nu} \propto \nu^{1.9 \beta / 6}$, with $\beta=1.6$ in the strong shock limit, for frequencies below a turnover $\nu_{\mathrm{t}}$, and is nearly flat $\left(S_{\nu} \propto \ln \nu_{\mathrm{t}}\right)$ from the turnover to the high energy cutoff. This theoretical synchrotron spectrum agrees notably well with the radio spectrum of the primary in Cyg OB2 No. 5, which presents a spectrum $\propto \nu^{0.54}$ between 4.8 and $8.4 \mathrm{GHz}$ (Waldron et al. 1998), that flattens at higher frequencies (Contreras et al. 1996). The value of $\nu_{\mathrm{t}}$, given by Eq. (45) of White (1985), is highly sensitive to small changes in the stellar parameters. For the values listed in Table 1 for the primary in Cyg OB2 No. 5 we obtain $\nu_{\mathrm{t}} \approx 10 \mathrm{GHz}$, in good agreement with the radio data.

The high-energy cutoff for charged particles accelerated in the vicinity of a star is (White 1985):

$E_{\max } \approx 1 \mathrm{GeV}\left(\frac{v_{\infty, 1}}{2500 \mathrm{~km} \mathrm{~s}^{-1}}\right)^{-1 / 2}\left(\frac{B_{*}}{\mathrm{G}}\right)\left(\frac{R_{*}}{35 R_{\odot}}\right)$,

which yields $E_{\max } \approx 2 \mathrm{GeV}$ for the primary in Cyg OB2 No. 5 (i.e. Lorentz factors $\gamma \sim 410^{3}$ ). This upper limit, imposed by geometric factors, is valid for both electrons and ions. However, in the case of electrons, radiative losses mainly due to IC scattering impose additional constraints (Chen \& White 1991), and hence the effective high-energy cutoff should occur at $\gamma \lesssim 10^{3}$ for them. Consequently, electron-induced gamma-rays in the base of the wind have energies $\lesssim 100 \mathrm{MeV}$ and are below EGRET energy threshold, as noticed by Chen \& White (1991).

Relativistic ions, on the contrary, can produce substancial gamma-ray luminosities through hadron interactions with the thermal wind nuclei and the subsequent $\pi^{0}$-decays (White \& Chen 1992). The resulting highenergy emission has a peak at $\sim 70 \mathrm{MeV}$ and then can significantly contribute to the gamma-ray flux detected by EGRET.

The calculation of the $\pi^{0}$ gamma-ray luminosity of the primary star in Cyg OB2 No. 5 has already been carried out by White \& Chen (1992), using the empirical formula for the $\pi^{0}$ production spectrum given by Stephens \& Badhwar (1981). The expected luminosity is $\sim 510^{34} \mathrm{erg} \mathrm{s}^{-1}$, similar to the IC luminosity of the colliding winds region.

\subsection{Summary of results}

In Table 2 we present a summary of the different contributions to the total gamma-ray luminosity of Cyg OB2 No. 5 in the EGRET energy range. The main contributions are IC emission from the colliding winds region and $\pi^{0}$-decay emission from the inner region of the primary stellar wind. These mechanisms can provide about a half of the inferred luminosity of the source 3 EG J2033+4118 (i.e. $\sim 1.310^{35} \mathrm{erg} \mathrm{s}^{-1}$ ). The observed gamma-ray flux could be explained if either (1) the mass illuminated by relativistic protons accelerated at the terminal shock of the wind is a factor $\sim 50$ larger than what we have assumed (i.e. if it is $\left.\sim 5000 M_{\odot}\right),(2)$ there are other gamma-ray sources within the EGRET $95 \%$ probability location contour or (3) there is an important contribution from the colliding winds region within the primary (i.e. in the close binary). This latter possibility cannot be quantitatively discussed because of the absence of observational constraints on the efficiency of the particle acceleration at the unresolved interacting region. The second possibility, namely that the EGRET detection could actually be a composed source, will be briefly discussed below.

\subsection{Other potential gamma-ray sources in the field}

There are, in addition to Cyg OB2 No. 5, other massive stars of the same association within the positional error box of 3EG J2033+4118, like stars No. 7, 8A, 9, and 11. These stars have similar parameters to the primary in Cyg OB2 No. 5 and are potential sources of $\pi^{0}$-decay gamma-rays. In particular, Cyg OB2 No. 8A and No. 9 are non-thermal radio emitters (Contreras et al. 1996; Waldron et al. 1998). White \& Chen (1992) have estimated the individual gamma-ray luminosities at $E>100 \mathrm{MeV}$ of these stars in $\sim 510^{34} \mathrm{erg} \mathrm{s}^{-1}$. The combined effect of these stars, plus the emission from the colliding winds region in Cyg OB2 No. 5, can easily explain the flux detected by EGRET. The absence of any other strong radio 
Table 2. Gamma-ray production in Cygnus OB2 No. 5 for EGRET energy range $(100 \mathrm{MeV}<E<20 \mathrm{GeV})$

\begin{tabular}{|c|c|c|c|}
\hline Region & Mechanism & $\begin{array}{l}\text { Expected luminosity } \\
(\mathrm{erg} / \mathrm{s})\end{array}$ & $\begin{array}{l}\text { Observed luminosity } \\
(\mathrm{erg} / \mathrm{s})\end{array}$ \\
\hline \multirow{3}{*}{ Winds collision } & Inverse Compton scattering & $\sim 810^{34}$ & \multirow{6}{*}{$\sim 2.410^{35}$} \\
\hline & Relativistic bremsstrahlung & $\sim 3.410^{30}$ & \\
\hline & Neutral pion decay & $\sim 5.210^{24}$ & \\
\hline Terminal shock & Neutral pion decay & $\sim 2.310^{32}$ & \\
\hline \multirow{2}{*}{ Base of the wind } & Inverse Compton scattering & - & \\
\hline & Neutral pion decay ${ }^{1}$ & $\sim 510^{34}$ & \\
\hline
\end{tabular}

1: White \& Chen (1992).

source in the field also supports this conjecture, which was advanced by White \& Cheng (1992) prior to the detection of non-thermal radio emission from Cyg OB2 No. 5 (see also Chen et al. 1996).

\section{Further comments}

\subsection{High-energy tail of the gamma-ray emision}

In the colliding winds region of Cyg OB2 No. 5 particles are accelerated at the shock front up to a high-energy cutoff that can be estimated assuming that the diffusion length is equal to the gyral radius. This yields,

$$
E_{\max }=1.310^{3} \mathrm{GeV}\left(\frac{v_{\mathrm{s}} B}{L_{2}}\right)^{1 / 2} r_{2},
$$

where $v_{\mathrm{s}}$ is the shock velocity in $\mathrm{km} \mathrm{s}^{-1}$. If $v_{\mathrm{s}}=v_{\infty, 1}$ and $B=B_{\mathrm{s}}$, we obtain $E_{\max } \sim 0.7 \mathrm{TeV}$. The IC highenergy emission, then, extends up to energies $\sim 20 \mathrm{TeV}$. The expected gamma-ray luminosity at $E>1 \mathrm{TeV}$ is $510^{30} \mathrm{erg} \mathrm{s}^{-1}$, which, at a distance of $1.8 \mathrm{kpc}$ gives a photon flux of $1.410^{-11} \mathrm{~cm}^{-2} \mathrm{~s}^{-1}$. This flux could be detected by planned gamma-ray ground arrays like VERITAS (Weekes et al. 2000).

\subsection{A few comments on the soft gamma-ray emission}

The colliding winds region can be an important source of soft gamma-ray photons. The IC flux density around $10 \mathrm{MeV}$ is $810^{-5} \mathrm{ph} \mathrm{cm}^{-2} \mathrm{~s}^{-1}$ and could be detected, in principle, by the Imager on Board INTEGRAL Satellite (IBIS), to be launched by the European Space Agency in April 2002 (Winkler \& Hermsen 2000). IBIS is a coded mask telescope designed to produce sky images in the $20 \mathrm{keV}-10 \mathrm{MeV}$ band with an angular resolution of 12 arcmin. It will also have a good spectral capability. The point source location accuracy for a relatively strong source will be around 1 arcmin. Consequently, IBIS will provide excellent diagnosis to test the models for gammaray production in Cyg OB2 No. 5 discussed in this paper. In particular, it will allow to measure the soft gammaray spectrum of the colliding winds and differentiate the emission of Cyg OB2 No. 5 from that produced in the neighboring stars, which are located at an angular distance $\geq 9$ arcmin. The main problem at these energies is the subtraction of the background galactic emission; notwithstanding, sufficiently accurate models of the diffuse radiation fields at low latitudes will be soon available (e.g. Bloemen et al. 2000). IC emission from the single stars should also be important at $\mathrm{MeV}$ energies. The hadronic gamma-ray component, however, should be observed with future higher energy telescopes, like the planned GLAST satellite, to be launched by NASA towards 2005 .

\section{Conclusions}

We have presented an analysis of the possible production of gamma-ray emission in the stellar system Cyg OB2 No. 5 taken into account the constraints imposed by recent high-resolution radio observations. We have estimated the gamma-ray luminosity generated by different types of mechanisms and compared them with the luminosity inferred from EGRET observations. We have found that about a half of the flux of the gamma-ray source 3EG J2033+4118 could arise from the colliding winds region (through IC interactions) and the base of the wind (through proton-proton interactions). It is possible, also, 
that the remaining flux comes from other nearby earlytype stars of the same OB association within the positional error box of the EGRET detection. We expect that forthcoming gamma-ray missions with good imaging capabilities, like the imminent ESA's INTEGRAL spacecraft, can clarify this point.

Acknowledgements. This work has been supported by the Argentine agencies CONICET (PIP 0430/98) and ANPCT (PICT 98 No. 03-04881), as well as by Fundación Antorchas. We thank Gerry Skinner and Gregor Rauw for valuable comments.

\section{References}

Abbott, D. C., Bieging, J. H., \& Churchwell, E. 1981, ApJ, 303, 239

Aharonian, F. A., \& Atoyan, A. M. 1996, A\&A, 917, 928

Bell, A. R. 1978a, MNRAS, 182, 147

Bell, A. R. 1978b, MNRAS, 182, 443

Bieging, J. H., Abbott, D. C., \& Churchwell, E. 1989, ApJ, 340,518

Blandford, R. D. 1980, ApJ, 238, 410

Blandford, R. D., \& Ostriker, J. P. 1978, ApJ, 221, L29

Bloemen, H., Hermsen, W., Collmar, W., et al. 2000, Proceedings of the Fourth INTEGRAL Workshop, ESA-SP, in press

Bohannan, B., \& Conti, P. S. 1976, ApJ, 204, 797

Cassé, M., \& Paul, J. A. 1980, ApJ, 237, 236

Chen, W., \& White, R. L. 1991, ApJ, 381, L63

Chen, W., White, R. L., \& Bertsch, D. L. 1996, A\&AS, 120, 433

Contreras, M. E., Rodríguez, L. F., Gómez, Y., \& Velázquez, A. 1996, ApJ, 469, 329

Contreras, M. E., Rodríguez, L. F., Tapia, M., et al. 1997, ApJ, 488, L153

Contreras, M. E., \& Rodríguez, L. F. 1999, ApJ, 515, 762

Dermer, C. D. 1986, A\&A, 157, 223

Dobashi, K., Bernard, J. P., Yonekura, Y., \& Fukui, Y. 1994, ApJS, 95, 419

Dobashi, K., Bernard, J. P., \& Fukui, Y. 1996, ApJ, 466, 282

Drury, L. O. 1983, Rept. Progr. Phys., 46, 473

Eichler, D., \& Usov, V. 1993, ApJ, 402, 271

Gehrels, N., Macomb, D. J., Bertsch, D. L., et al. 2000, Nature, 404, 363

Hall, D. S. 1974, Acta Astron., 24, 69
Hartman, R. C., Bertsch, D. L., Bloom, S. D., et al. 1999, ApJS, 123, 79

Herbig, G. H. 1967, PASP, 79, 502

Lamers, H. J. G. L. M., \& Cassinelli, J. P. 1999, Introduction to Stellar Winds (Cambridge University Press, UK)

Leitherer, C., Hefele, H., Stahl, O., \& Wolf, B. 1982, A\&A, 108, 102

Lucy, L. B. 1982, ApJ, 255, 286

Lucy, L. B., \& White, R. L. 1980, ApJ, 241, 300

Maheswaran, M., \& Cassinelli, J. P. 1992, ApJ, 386, 695

Miralles, M. P., Rodríguez, L. F., Tapia, M., et al. 1994, A\&A, 282,547

Paredes, J. M., Mart, J., Rib, M., \& Massi, M. 2000, Science, 288,2341

Persi, P., Tapia, M., Rodriguez, L. F., et al. 1990, A\&A, 240, 93

Pollock, A. M. T. 1985, A\&A, 150, 339

Rauw, G., Vreux, J.-M., \& Bohannan, B. 1999, ApJ, 517, 416

Romero, G. E., Benaglia, P., \& Torres, D. F. 1999, A\&A, 348, 868

Romero, G. E., Torres, D. F., Benaglia, P., et al. 2000, Proceedings of the Fourth INTEGRAL Workshop, ESA-SP, in press

Spitzer, L. 1998, Physical Processes in the Interstellar Medium (Wiley, New York)

Stephens, S. A., \& Badhwar, G. D. 1981, Ap\&SS, 76, 213

Torres, D. F., Romero, G. E., Combi, J. A., et al. 2000 [astroph/0007464]

Vacca, W. D., Garmany, C. D., \& Schull, J. M. 1996, ApJ, 460, 914

Völk, H. J., \& Forman, M. 1982, ApJ, 253, 188

Uesugi, A., \& Fukuda, I. 1982, Revised Catalogue of Stellar Rotational Velocities (Kyoto University, Kyoto)

Walborn, N. 1973, ApJ, 180, L35

Waldron, W. L., Corcoran, M. F., Drake, S. A., \& Smale, A. P. 1998, ApJS, 118, 217

Weekes, T. C., Bradbury, S. M., Bond, I. H., et al. 2000, in Proceedings of the Fifth Compton Symposium, ed. M. L. McConnel, \& J. M. Ryan (AIP, New York), 637

White, R. L. 1985, ApJ, 289, 698

White, R. L., \& Chen, W. 1992, ApJ, 387, L81

White, R. L., \& Chen, W. 1995, in IAU Symp. 163, ed. K. A. van der Hucht, \& P. M. Williams (Kluwer, Dordrecht), 438

Winkler, C., \& Hermsen, W. 2000, in Proceedings of the Fifth Compton Symposium, ed. M. L. McConnel, \& J. M. Ryan (AIP, New York), 676

Zhekov, S. A., \& Skinner, S. L. 2000, ApJ, 538, 808

Zhang, L., Zhang, Y. J., \& Cheng, K. S. 2000, A\&A, 357, 957 